\title{
CRÍTICA A LAS CIENCIAS AGRARIAS
}

\author{
David Vásquez Cardona \\ Ingeniero Agrónomo Integrante del Centro de Pensamiento Latinoamericano Raíz-al \\ dvsaudadeazul@gmail.com
}

\author{
“...La vida productiva es la vida de \\ La especie. Es la vida engendradora de vida. \\ El tipo de actividad vital lleva en sí todo el carácter \\ de una species, su carácter genérico, y la \\ actividad libre y consciente es el carácter \\ genérico del hombre".
}

Karl Marx Manuscritos de 1844.

Manizales, 2008-10-30 (Rev. 2008-11-28)

\begin{abstract}
RESUMEN
La crisis ambiental desatada en el actual modo de producción cuestiona el quehacer de las ciencias agrarias, dado que las tecnologías por ellas elaboradas han devenido en el deterioro de la vida en los ecosistemas. Tales desarrollos se realizan bajo el argumento del ejercicio científico que sustenta un objetivo para imponer su saber violento que busca la dominación de la naturaleza más que su explicación y la ordenación de la misma en teorías, cual es el quehacer científico. Sobre esta lógica, dichas ciencias especializadas dentro de los diversos campos de la división del trabajo han tenido por principio el monocultivo, forma característica de la agricultura desarrollada dentro del proceso de producción de mercancías de la sociedad burguesa, razón por la cual sus logros no comprenden las dinámicas de la vida en los ecosistemas, acelerando la entropía, generando con ello un deterioro de la vida en el planeta, lo que sólo puede ser superado en la medida en la que el hombre desde su trabajo, que le da formas a la sustancia de la naturaleza, se reconcilie con ella por medio de su acción consciente.
\end{abstract}

\section{PALABRAS CLAVE}

Crisis ambiental, ciencias agrarias, monocultivo, entropía, vida.

\section{CRITICISM TO AGRARIAN SCIENCES}

\begin{abstract}
The environmental crisis caused by the current mode of production criticizes the labor of Agrarian Sciences, due to the fact that they produced technologies that have deteriorated life in ecosystems. These developments have been carried out claiming to be exercising their scientific labor supporting the goal to impose their violent knowledge, which seeks to control nature, instead of its explanation and organization in theories, their true scientific duty. Based on this logic, said sciences specialized in the diverse sectors of the division of labor aimed at single-crop farming, the
\end{abstract}


characteristic form of agriculture developed within the goods production process of bourgeois society. Therefore, the achievements of Agrarian Sciences don't address the dynamics of life in ecosystems, but rather accelerate entropy, and thus deteriorate life on the planet; this can only be overcome if humans by their work, which shapes nature, reconcile with nature through their conscious action.

\section{KEY WORDS}

Environmental crisis, Agrarian Sciences, single-crop farming, entropy, life.

La pregunta que orienta la investigación que se despliega en este trabajo nace de la crisis ambiental que soporta el planeta tierra ${ }^{1}$, y de la manera en la que es tratada dicha crisis en las facultades de agronomía, donde es tomada como una molestia menor del ejercicio profesional y no como fuente de indagación permanente como corresponde, pues compete al objeto de estudio de las ciencias agrícolas -la agricultura- tratar con los elementos propios de los ecosistemas y sus problemas, que son hoy de interés capital para los seres humanos, pues en ellos está comprometida la existencia de la vida.

Se establecieron así, desde la reflexión crítica, las siguientes preguntas: Si la agronomía y las ciencias agronómicas son ciencias ${ }^{2}$, es decir, son construcciones teóricas que ordenan la realidad para transformarla de manera racional por medio del ejercicio técnico ¿porqué sus desarrollos atentan contra la vida de los ecosistemas y, por lo tanto, contra la vida del hombre? ¿Es que acaso los desarrollos científicos de la agronomía no han logrado ordenar los hechos propios del ejercicio agrícola, razón por la cual la aplicación de la colección de datos desordenados por medio de los logros técnicos, son los causantes de que en cada paso la producción agrícola socave la vida natural y humana?

Para resolver dichas preguntas se decidió mirar la historia de la agronomía, buscando en su movimiento los elementos propios de sus desarrollos, destacando en la exposición los diversos problemas que detenta. Hemos encontrado así que la agronomía surge a inicios del siglo XIX, momento en el que el mundo rural de Europa y los Estados Unidos de Norteamérica se encontraba en su primera revolución agrícola ${ }^{3}$. La conformación de los primeros "institutos agronómicos" ubicados en los campos con granjas experimentales, son producto de la inspiración de dicha revolución ${ }^{5}$. Dadas las necesidades crecientes del sector, a mediados del mismo siglo en Alemania los institutos son trasladados a las universidades $\mathrm{y}$, en Francia, se fundan las primeras escuelas de agronomía ${ }^{6}$. Los nuevos conocimientos producidos son aplicados directamente en la agricultura, lo que da origen a la segunda revolución agrícola que tiene como característica la introducción del saber científico en la dinámica productiva, lo que sólo vendría a reafirmar la primera revolución dado que el modelo tecnológico que devino de la misma profundiza sobre los elementos de la primera.

Los diversos estudios en química agrícola, fisiología vegetal, genética, microbiología y mecánica, abren el escenario para la aplicación del modelo tecnológico que se mantiene hasta nuestros días, el cual tiene como principio la aplicación de fertilizantes de alta solubilidad, la utilización de semillas mejoradas para las siembras ${ }^{7}$, la mecanización de las labores agrícolas, la utilización de sistemas de regadío y la aplicación de biocidas para el control de arvenses, hongos e insectos ${ }^{8}$. 
Los más diversos estudios realizados desde el nacimiento de la agricultura han estado limitados a la forma agrícola del monocultivo ${ }^{9}$, pues el nacimiento de la agronomía corresponde con el régimen de producción de mercancías de la sociedad burguesa, que determina la especialización de las unidades y el crecimiento de la división del trabajo, pues el propietario de la tierra realiza la explotación de la misma de acuerdo a los beneficios que pueda extraer de la producción, atendiendo la calidad del terreno, las comunicaciones, la cuantía de capital, la extensión de su propiedad, etc. ${ }^{10}$. La agronomía aparece como manifestación propia del mundo burgués, por lo tanto, sus desarrollos no pueden más que corresponder al mismo, y sus logros limitarse, en un primer momento, a la producción de mercancías, siendo la mercancía, la "forma más general y rudimentaria de la sociedad burguesa."

Como bien nos lo ha indicado Marx, los modos de producción de una sociedad corresponden con los modos de vida de la misma ${ }^{12}$, por lo que los estudios agrícolas que hacen parte de los avances en el conocimiento de la sociedad burguesa no están aislados de la forma en la que ella produce la vida, que se manifiesta en el régimen de producción de mercancías, sino que por el contrario son su producto y permiten de manera dialéctica su reproducción.

El conocimiento agrícola restringido a la forma agrícola del monocultivo, no ha podido comprender las relaciones diversas de los ecosistemas, para integrarlas, en un primer momento, en los estudios e investigaciones del objeto de conocimiento y, en un segundo momento, en las dinámicas productivas; por lo que sus desarrollos técnicos son producto de la aplicación desordenada de una serie de hechos empíricos que, a cada paso, socavan la vida de la naturaleza y del hombre.

Se manifiesta de esta manera el divorcio del intelecto del hombre con el conocimiento de la naturaleza del objeto. El objeto y su naturaleza quedan sometidos al sujeto que establece una relación patriarcal sobre las cosas, las cuales deben estar al servicio de la vida del hombre. Así, el sujeto envuelto en un conocer violento toma para sí la vida de la naturaleza, sin comprender que ella es su "cuerpo inorgánico", con la cual debe permanecer para no morir, y en los abusos contra ella cometidos producto del extrañamiento, de la enajenación, el hombre no sólo atenta contra la vida natural sino contra su propia vida, pues "la afirmación de que la vida física y espiritual del hombre se halla entroncada con la naturaleza, no tiene más sentido que el que la naturaleza se halla entroncada consigo misma, ya que el hombre es parte de la naturaleza"13.

Lo que los hombres quieren en el conocimiento de la naturaleza es la forma de utilizarla para su sometimiento integral. Este modo violento del proceder convierte el poder y el conocer en sinónimos, lo que está presente en la sociedad burguesa desde el empirismo de Bacon y desde las pretensiones de Lutero, para quienes no importa lo que los hombres llaman verdad sino el procedimiento eficaz. Bacon nos dice que: "el verdadero fin y tarea de la ciencia" reside no en "discursos plausibles, edificantes, dignos o llenos de efecto, o en supuestos argumentos evidentes, sino en el empeño y en el trabajo, y en el descubrimiento de detalles antes desconocidos para un mejor equipamiento y ayuda en la vida"14.

El conocimiento, de esta forma, renuncia al deseo de revelación, queda relegado a la descripción de hechos y de observaciones propias de la experiencia y, la noción empirista que conduce el "aparato teórico" del iluminismo, hace que la reconciliación sujeto y el objeto pensada por Hegel y Marx se disuelva en el aire. Como bien lo exponen Horkhaimer y Adorno: 
"Sujeto y objeto quedan ambos anulados... La ecuación espíritu y mundo se disuelve finalmente, pero sólo de tal modo que ambos términos se reducen recíprocamente. Lo que parece un triunfo de la racionalidad objetiva, la sumisión de todo lo que existe al formalismo lógico, es pagado mediante la dócil sumisión de la razón a los datos inmediatos... La entera pretensión del conocimiento es abandonada. Ella no consiste sólo en percibir, clasificar y calcular, sino justamente en la negación determinada de lo inmediato"15.

En los orígenes de la ciencia encontramos que ésta busca comprender, explicar y ordenar la realidad por medio de los conceptos ${ }^{16}$, pretensión que se disuelve en el "aparato teórico" del mundo burgués, donde reina el empirismo y la observación de los hechos concretos, que buscan la dominación del cuerpo natural de las cosas.

Cuando damos cuenta del divorcio entre objeto y sujeto en las ciencia agrícolas aparece inmediatamente la posibilidad de su superación, pues la conciencia lograda desarrolla un camino teleológico que tiene por principio encontrar los elementos más íntimos de los ecosistemas sobre los cuales construir los nuevos modelos de conocimiento y aplicaciones propiamente agrícolas. En esta medida se abre todo un campo de investigación, en el cual ya existe un recorrido por parte de los estudios agroecológicos, que hoy vienen dando los primeros pasos para la reconciliación del hombre con la naturaleza ${ }^{17}$.

El lugar de inicio para integrar los principios ecológicos con la agricultura están dados por la ecología quien nos ha "enseñado que las poblaciones no sólo evitan los extremos suicidas, sino que evolucionan más bien hacia la regulación de su densidad a un nivel pronunciadamente por debajo de la asíntota o capacidad de soporte, que podría conseguirse si todos los recursos en materia, de energía y espacio se utilizaran cabalmente"18. A diferencia de lo que se piensa en la forma agrícola del monocultivo -que elimina la vida que circunda los cultivos, considerándola como competencia y no como productora de reacciones simbióticas en beneficio de la vida de los mismos cultivos ${ }^{19}$ - son los diversos componentes vivos y abióticos del ecosistema los que determinan la posibilidad del equilibrio energético, de producción y reproducción de la vida y, por lo tanto, de los cultivos; entre más diverso sea un ecosistema más estable es y entre más simple sea, como lo es el paisaje monotemático de la agricultura burguesa, más inestable, el cual demanda una mayor cantidad de energía externa para permitir la producción ${ }^{20}$.

La termodinámica nos ha enseñado que el proceso entrópico de los sistemas vivos se acelera en la medida en la que no se reutilizan los excedentes de la producción $^{21}$, lo que significa el deterioro permanente de la naturaleza en el planeta, razón por la cual es de vital importancia considerar las relaciones energéticas en los desarrollos tecnológicos para el alcance de un nuevo modo de producción que engendre vida -a partir de la actividad consciente y libre de los hombres- y no muerte, como viene ocurriendo desde la actividad enajenada de éstos, propia del actual modo de producción. Es así que la relación termodinámica entre la estática y dinámica presente en todos los ecosistemas es fundamental para considerar las aplicaciones tecnológicas ${ }^{22}$. Este enfoque abre un espacio de investigación sobre los potenciales productivos de los diferentes ecosistemas considerando la relación antes señalada, lo que ha venido siendo analizado por diversos investigadores en América Latina ${ }^{23}$.

Pensar de esta forma la agronomía requiere compromiso de hombres y mujeres que, motivados por su voluntad consciente, promuevan el desarrollo de estudios que integran la producción agrícola con la dinámica misma de la naturaleza a la 
que, evidentemente, no es extraño el hombre, sino que por el contrario pertenece; lo anterior es necesario para que los modelos tecnológicos que transforman la realidad natural estén guiados por la razón que ha aprehendido lo real, siendo la técnica la manifestación de la razón en la realidad y, por lo tanto, la forma en la que lo racional se hace real.

Para el logro de la superación del divorcio entre el intelecto humano y la naturaleza, se requiere que el espíritu que guía la civilización en su movimiento de transformar conservando, supere los modos de vida que se imponen en el mundo burgués, los cuales están determinados por el régimen de producción de mercancías ${ }^{24}$, donde el hombre, como hemos indicado, se encuentra enajenado de la naturaleza y de su propio trabajo ${ }^{25}$. Hacer conciencia del extrañamiento que se impone a los hombres producto de las relaciones humanas establecidas por la propiedad privada ${ }^{26}$, la división del trabajo y el modo de producción de la actual sociedad -que hacen parte de un mismo régimen- es el inicio de las bodas del hombre con la naturaleza, pues es el principio de la emancipación de los modos de vida de la actual sociedad, con lo cual deben estar comprometidos aquellos hombres que, guiados por la conciencia, comprometen la voluntad, lo que implica una actitud ética que busca reafirmarse en la vida de la sociedad y de la naturaleza.

Esperamos que lo expuesto logre con el cometido de iniciar la demolición de la agronomía, para resarcirla de sus escombros y ponerla a la altura del más alto fin humano: la conquista de la libertad.

\section{REFERENCIAS BIBLIOGRÁFICAS}

- Bejarano, Antonio. Economía y poder, la SAC y el desarrollo agropecuario colombiano, 1871-1984, Fondo editorial CEREC, Sociedad de Agricultores Colombianos, 1985.

- Bejarano, Antonio. Ensayos de Historia Agraria en Colombia, Fondo Editorial Cerec, Bogotá, 1987

- Declaración de la Conferencia de las Naciones Unidas sobre el Medio Ambiente, Reunidas en Estocolmo del 5 al 16 de Junio de 1972.

- Fukoka, Masanobu. La Senda Natural del Cultivo Regreso al Cultivo Natural. teoría y práctica de una filosofía verde, Terarion, Valencia, 1995.

- Horkheimer, M.; Adorno, W. Theodor. Dialéctica de la ilustración Fragmentos filosóficos, introducción y traducción: Juan José, Sánchez. 3a. edición, Trotta, 1998.

- Kaustky, Karl. La Cuestión Agraria, Editorial Latina, Bogotá, 1983.

- Marx, K. El Capital, Crítica de la Economía política, Fondo de Cultura Económica, Bogotá, 1976

- Marx, K. Manuscritos Económico-filosóficos de 1844, Tra. Cast. Wenceslao Roces, Editorial Grijalbo, México, 1968.

- Marx, K., Engels, F. La ideología Alemana, Fondo editorial progreso, Bogotá, 1975.

- Mesa, Darío. Tesis sobre Feuerbach, de Karl Marx, Ensayos sobre teoría sociológica (Durkheim, Weber y Marx), Universidad Nacional, Bogotá, 2002.

- Odum, Eugene P. Ecología,3a. edición, Editorial Interamericana, 1985.

- Restrepo, Jairo. Sol, Termodinámica y Agricultura, San José, 1994.

- Restrepo, Jairo. Venenos del invento al uso y de la muerte a la vida, Habana, Agosto de 1996.

- Taton, Rene. Historia general de las ciencias, Vol. 3, Ediciones Destino, Barcelona, 1973. 


\section{NOTAS}

1. Hace más de 30 años dicha crisis fue reconocida por la Declaración de la Conferencia de las Naciones Unidas sobre el Medio Ambiente, Reunidas en Estocolmo del 5 al 16 de Junio de 1972. donde se destaca que: "A nuestro alrededor vemos multiplicarse las pruebas del daño causado por el hombre en muchas regiones de la Tierra: niveles peligrosos de contaminación del agua, el aire, la tierra y los seres vivos; grandes trastornos del equilibrio ecológico de la biosfera; destrucción y agotamiento de recursos insustituibles y graves deficiencias, nocivas para la salud física, mental y social del hombre, en el medio por el creado, especialmente en aquel en que vive y trabaja".

2. Antonio Bejarano señala que lo que se ha catalogado como agricultura científica - ciencias agropecuarias no podría entenderse "si no las viéramos como el resultado de la confluencia simultanea del desarrollo de las ciencias y de las preocupaciones por la técnica." Bejarano, Antonio. Ensayos de Historia Agraria en Colombia, Fondo Editorial Cerec, Bogotá, 1987, p. 115.

3. Los principales componentes de la primera revolución agrícola pueden resumirse en la gradual eliminación del barbecho, sustitución por continuas rotaciones de cultivos -cuestión que desaparecería rápidamente en la medida en la que se desarrolló la especialización de las unidades de producción agrícola- introducción o extensión de nuevos cultivos, mejora de herramientas de uso tradicional, selección de semillas, crías de ganado de manera estabulada, lo que permitió la utilización de nuevas tierras para los cultivos y las mejoras productiva de las mismas con la aplicación del estiércol del ganado, aumento del uso de animales para el trabajo agrícola. Bejarano, Antonio. Economía y poder, la SAC y el desarrollo agropecuario colombiano, 1871-1984, Fondo editorial CEREC, Sociedad de Agricultores Colombianos, 1985, p 116. Igual ver Kaustky, Karl. La Cuestión Agraria, Editorial Latina, Bogotá, 1983, p. 37 y ss.

4. Los primeros institutos agronómicos que se fundan son los de Celle, en 1802, y de Moglin, en 1804; otros institutos surgirían en los años siguientes estando en primer nivel el de Hohenheim en Wuttemberg, 1918. Kaustky, Karl. Op. cit., p. 59.

5. Un ejemplo de ello lo encontramos en la obra de Thaer, quien después de analizar el proceso la revolución agrícola que sufrió Inglaterra, promovía en su obra la conformación de los institutos agrícolas en Alemania con el objeto de introducir la ciencia en la agricultura alemana. Op. cit., p. 59.

6. Las primeras escuelas de estudios agrícolas que se establecen en Francia son las escuelas de Grignon, en 1848, y Ruennes, en 1849, que ejercieron una gran influencia; de igual forma, en 1848, lo que había sido la escuela politécnica de ciencias físicas, químicas y naturales, adopta el nombre de "Instituto Nacional Agronómico", aunque más tarde es suprimido. Bejarano, Antonio. Ensayos de Historia Agraria en Colombia, Fondo Editorial Cerec, Bogotá, 1987, p. 127.

7. Las semillas mejoradas en la aplicación agrícola comienzan en 1880 , con el estudio estadístico de 170 variedades de tubérculos. En un momento en el que se tenía en el olvido los estudios sobre la herencia logrados por Gregor Mendel. Bejarano, Antonio. Economía y poder. La SAC y el desarrollo agropecuario colombiano 1871 -1874, Fondo Editorial Cerec, Bogotá, 1985, p. 95. 
8. Si bien los estudios sobre patógenos en los cultivos comienzan en 1863, con Bary -quien descubre el parasitismo de los hongos en los cultivos- y las aplicaciones para el control de los mismos inician con Millardet en 1882 -quien descubre el caldo Bordeles- las aplicaciones masivas de biocidas sólo se dan hasta 1940, después de haber sido utilizados en la guerra y reconvertidos a la agricultura por parte de las industrias productoras de los mismos entre las que se encuentra la Bayer. En lo concerniente al estudio de los patógenos ver Taton, Rene. Historia general de las ciencias, Vol. 3, Ediciones Destino, Barcelona, 1973, p. 600-601; y en lo que tiene que ver con el uso de los venenos ver Restrepo, Jairo. Venenos del invento al uso y de la muerte a la vida, Habana, Agosto de 1996, p. 63.

9. Cuando nos referimos a la forma del monocultivo estamos indicando la forma en la que la agricultura aparece en una época de la historia, a la cual se debe un desarrollo propio que le da contenido.

10. Para 1894, la especialización llegaba a tal punto que en Inglaterra se hacían subdivisiones en las explotaciones dentro de una misma especie animal. Así, en la lechería distinguen la producción de leche fresca para la venta, de la utilizada para hacer mantequilla y de la requerida para hacer queso, utilizando en cada explotación razas distintas de animales y diferentes métodos de cría. Kaustky, Karl, Op. cit. p. 43.

11. Marx, K. El Capital, Crítica de la Economía política, Fondo de Cultura Económica, Bogotá, 1976, p. 46.

12. Al respecto mirar Marx, K., Engels, F. La ideología Alemana, Fondo editorial progreso, Bogotá, 1975, p. 22.

13. Marx, K. Manuscritos Económico-filosóficos de 1844, Tra. Cast. Wenceslao

Roces, Editorial Grijalbo, México, 1968, p. 80.

14. Lo anterior se puede leer en el concepto del lluminismo en Horkheimer, M.; Adorno, W. Theodor. Dialéctica de la ilustración Fragmentos filosóficos, introducción y traducción: Juan José, Sánchez. 3a. edición, Trotta, 1998, p. 6.

15. Op. cit., p. 80.

16. Este ejercicio comienza con la negación de la experiencia sensible, es decir, parte de los hechos singulares de los fenómenos, y se eleva sobre ellos, por medio de la abstracción que realiza el intelecto para ordenarlos de manera conceptual, a los cuales debe volver para corroborar el poder del concepto. Esto lo encontramos en Pascal cuando nos dice que "el principio de la geometría consiste en partir de los hechos singulares para elevarnos de ellos hasta los principios generales y volver de los principios generales a los hechos singulares para ver si esos principios están debidamente construidos, elaborados, etc." Léase los principios generales Pascal como conceptos. Mesa, Darío. Tesis sobre Feuerbach, de Karl Marx, Ensayos sobre teoría sociológica (Durkheim, Weber y Marx), Universidad Nacional, Bogotá, 2002, p. 89-90.

17. A partir de una noción ecológica de la producción, se han venido desarrollando importantes aportes entre los que se encuentra: los trabajos de Miguel Ángel Altieri sobre policultivos y biodiversidad (2000); el manejo ecológico del suelo por parte Ana Primavesi, (1980); la teoría de la trofobiosis elaborada por Francis Chaboussou, (1969); la producción de fertilizantes dinamizados por 
microorganismos de origen no sintético, por parte de Jairo Restrepo, (1999), partiendo de la utilización de los principios de la transmutación de los elementos químicos a partir de la actividad biológica, señalada por Kervran (1962) y Soleil (1994); los aportes en termodinámica y economía por parte de Roegen, Georgescu (1996); los análisis sobre ecología y capitalismos entre los que se encuentran Anndré Gorz (1991), James O'Connor (2001), y otros.

18. Odum, Eugene P. Ecología 3a. edición, Editorial Interamericana, 1985, p 218.

19. Odum acertó al decir que: "la aceptación generalizada de la idea de Darwin de la supervivencia de los mejor adaptados, ha dirigido la atención hacia los aspectos competitivos de la naturaleza y como consecuencia de ello, la importancia de la cooperación entre especies ha sido tal vez subestimada". Op. cit., p. 253.

20. Los modelos agrícolas modernos son tan inconsistentes desde su propia lógica de acumulación de capital que es el objetivo básico del monocultivo que granjeros de los Estados Unidos que trabajan de 500 a 700 acres tienen menos ingresos netos que los granjeros japoneses que explotan de 3 a 5 acres. Al respecto ver Fukoka, Masanobu. La Senda Natural del Cultivo Regreso al Cultivo Natural. teoría y práctica de una filosofía verde, Terarion, Valencia, 1995, p. 43.

21. El proceso de producción agrícola, como todo el proceso de producción de mercancías, consiste materialmente en una transformación de baja en alta entropía, es decir, en desechos. Los desechos y excedentes de la producción se deben reutilizarse para disminuir el efecto entrópico de los ecosistemas.

22. La diferencia de temperatura y, por lo tanto, de energía entre los ecosistemas determina la relación entre dinámica y estática de los procesos bióticos, abióticos y de su relación.

23. Restrepo, Jairo. Sol, Termodinámica y Agricultura, San José, 1994. p.7. Donde el autor destaca la importancia del aporte de Juquira Candiru, sobre este tema.

24. La forma social del trabajo que los hombres realizan los unos para los otros toma una forma de cualquier modo que lo hagan, lo que en el mundo burgués aparece en la forma de mercancíaque es "la forma más general y rudimentaria de la sociedad burguesa", en la cual el valor de cambio dado por el trabajo, es apropiado por el dueño de los medios de producción, el cual haciendo valer su propiedad privada se toma para sí el trabajo privado del obrero, el cual se extraña de su trabajo y de la naturaleza con que realiza su trabajo, del que depende para subsistir de un modo meramente físico. Para leer la forma de la mercancía mirar Marx, K. El Capital, Crítica de la Economía política, Fondo de Cultura Económica, Bogotá, 1976, p. 46.

25. La enajenación de la naturaleza en la sociedad burguesa aparece en un doble sentido, el primero es el que se ha destacado en la exposición, donde el hombre al tomar la naturaleza para dominarla la desconoce en la renuncia que realiza del revelamiento de las cosas, privilegiando las observaciones empíricas que permiten el procedimiento, el facto, la dominación, lo que conduce al deterioro ambiental. La segunda es la destacada por Marx donde el obrero se extraña de su "cuerpo inorgánico", la naturaleza, la cual entrega al dueño de la producción, desconociendo en la transformación de la naturaleza su expresión en ella a partir de su actividad vital. La enajenación de su trabajo -el que entrega a otro hombre- y de la naturaleza, es un extrañamiento con respecto a otro hombre por lo cual pierde su 
condición genérica, su condición como especie. Marx, K. Manuscritos Económicofilosóficos de 1844, tr. cast. Wenceslao Roces, Editorial Grijalbo, México, 1968, p. 84.

26. Marx destaca de manera brillante que si bien el trabajo enajenado fue extraído de la economía política como resultado del movimiento de la propiedad privada, "la propiedad privada es el resultado, la consecuencia necesaria del trabajo enajenado, de la relación externa del obrero con la naturaleza y consigo mismo. (...) aunque la propiedad privada aparezca como el fundamento, como la causa del trabajo enajenado, es más bien una consecuencia de éste, del mismo modo que los dioses no son, en su origen, la causa, sino el efecto del extravío de la mente del hombre. Más tarde la relación se convierte en una relación de interdependencia”. Queda así establecido que, la relación entre los modos de vida y los modos de producción están definidos por una relación de interdependencia, es decir, que se corresponden. Superar la enajenación pasa necesariamente por la conciencia de los modos de vida de la sociedad burguesa, la cual debe guiar la acción de la voluntad en la transformación. 